\title{
Block Structure of a Number of the Integers Prime
}

\author{
P.M. Mazurkin ${ }^{*}$ \\ Doctor of Engineering Science, Academician of RANS, member of EANS, Volga Region State Technological University, Russia \\ *Corresponding author: kaf_po@mail.ru
}

\section{Received July 17, 2014; Revised August 01, 2014; Accepted August 10, 2014}

\begin{abstract}
Binary decomposition of numbers forms geometrical blocks. They depend on approach of a prime or whole prime number to values of the two in the degree equal to number from a natural row. As a result there is a strict geometry among prime or whole prime numbers in the form of block structure. This structure receives distinctive signs and harmonicas in positive part of a number of the whole prime numbers are shown. Statistically from a natural numbers regularities of growth of power of the left and right reference points, as borders previous and the subsequent from values of the two in degree, at blocks increasing on length among the whole prime numbers are proved.
\end{abstract}

Keywords: whole prime numbers, positive part, binary representation, block structure, reference point, distinctive signs, wave regularities

Cite This Article: P.M. Mazurkin, "Block Structure of a Number of the Integers Prime." Applied Mathematics and Physics, vol. 2, no. 4 (2014): 135-145. doi: 10.12691/amp-2-4-3.

\section{Introduction}

Mathematicians counted up to our publications [1-6] that known ranks of prime numbers don't possess geometrical properties. A Gaussian row and prime numbers special ranks seemed a senseless heap of numbers in the form of unclear "ladder" on which the leader in one of a series of movies on mathematics stories climbs up. The strong psychological barrier in understanding of geometrical sense arose when young Gauss cleaned from presented to it to 15-year age of the table of prime numbers unit. Thereby the system of binary notation from the beginning of a complete series of prime numbers was completely cut.

Our researches showed that the nature "considers" in a binary numeral system, and the person - in decimal system. Therefore it appeared that any number of prime numbers can be curtailed in a binary numeral system [4]. And only in this case there is a geometry as most Gaussian ladder, so especially its steps without the triangular basis of this ladder (a gain of prime numbers [2,6]).

Article purpose - to show block structure of a number of the whole prime numbers on its positive part and to give regularities of quantity (power) of prime numbers.

\section{Quantization of a prime number}

Any natural or an integer, including and prime numbers, it is possible to present as quantized in a binary numeral system a discrete signal. But quantization and formation (generation) of prime numbers while is unknown process.

At quantization all area of values of a signal breaks into the levels which quantity has to be presented in numbers of the set word length. Distances between these levels is called as a quantization step, and it for not rarefied (not specific) a row natural or integers is equal 1 . The whole and positive prime numbers (further prime numbers - PN) are specific ranks, and with while to unknown variables with increase of a number of PN by a quantization step (length of the block of PN).

The number of these levels (categories of a binary numeral system) depends on the power of a quantized number of prime numbers. It is known long ago that for the numbers growing on rather simple laws, for example, on degrees of the two, of course, it would be ridiculous to search for the copy surpassing all known. For simple same number attached great efforts to do just that. Prime numbers as ranks were exposed only to factorization, i.e. decomposition on multipliers and numbers with big degrees of the two. However factorization limits at a number of prime numbers still aren't found.

Our results show that it is necessary to do decomposition on composed, and this composed will be from binary representation number from a decimal numeral system. Such approach allows to return to mathematicians directly to studying of ranks, instead of to be engaged in laws of growth of quantity of prime numbers in categories of a decimal numeral system.

Here this hobby for factorization as we believe, and didn't allow mathematics to apply to us binary system to the analysis not only multipliers of any prime number, but also quantized in a binary numeral system composed from any natural or an integer, and, in particular, and any prime number. Decomposition on composed of a binary numeral system gives advantages in presentation of geometry.

On hierarchy we will consider some main properties inherent in prime numbers.

1. Any Prime number known in the Gaussian series $a(n)=2,3,5,7,11, \ldots$ contains bits $i_{2}=1,2, \ldots$ of the binary 
system and therefore has after decomposition of the components of components

$$
a_{i_{2}}(n)=2^{i_{2}-1}
$$

where $a_{i_{2}}(n)$ - quantized on levels $i_{2}$ of a binary numeral system part of a prime number.

2. Any prime number or the whole prime number is equal to the sum of components from a binary numeral system, taking into account the incidence matrix

$$
a(n)=\sum_{i_{2}=1}^{\infty} \xi\left(i_{2}, n\right) a_{i_{2}}(n)=\sum_{i_{2}=1}^{\infty} \xi\left(i_{2}, n\right) 2^{i_{2}-1}
$$

where $\xi\left(i_{2}, n\right)$ - an incidence matrix, and always $\xi\left(i_{2}, n\right)=0 \vee 1$.

\section{Block of prime numbers}

Computing experiments showed that the power of a row needs to be accepted proceeding from completeness of blocks of a matrix of incidence. For an example we take the block № 11 of positive part of a number of prime numbers [4] with the fragment having parameters: $n=(173,309), a(n)=(1031,2039), i_{2}=(1,11)$.

Calculations for the block № 11 are given in Table 1 and executed on a formula

$$
z_{2}=a_{1}-a_{2} \cos \left(\pi a(n) /\left(a_{3}+a_{4} a(n)^{a_{5}}\right)-a_{6}\right)
$$

where $z_{2}$ - the binary number changing ranging from 0 to 1 as a real number, $a(n)=2,3,5,7,11, \ldots$ - Gaussian number of prime numbers, $a_{1} \ldots a_{6}$ - parameters of

\begin{tabular}{|c|c|c|c|c|c|c|c|c|c|}
\hline \multirow{2}{*}{$\begin{array}{c}\text { Category } \\
i_{2}\end{array}$} & \multirow{2}{*}{$\begin{array}{c}\text { Part } \\
a_{i_{2}}(n)\end{array}$} & \multirow{2}{*}{$\begin{array}{c}\text { Average } \\
\text { statistical. } \bar{Z}_{2 \phi}\end{array}$} & \multicolumn{6}{|c|}{ Parameters of statistical model (3) of binary number } & \multirow{2}{*}{$\begin{array}{l}\text { Correlation } \\
\text { coefficient } r\end{array}$} \\
\hline & & & $a_{1}$ & $a_{2}$ & $a_{3}$ & $a_{4}$ & $a_{5}$ & $a_{6}$ & \\
\hline 1 & 1 & 1 & 0.5 & -0.5 & 0 & 0 & 0 & 0 & 1 \\
\hline 2 & 2 & 0.51825 & 0.5 & 0.5 & 2 & 0 & 0 & 1.59217 & 1 \\
\hline 3 & 4 & 0.51825 & 0.5 & 0.70711 & 4 & 0 & 0 & 1.57080 & 1 \\
\hline 4 & 8 & 0.53285 & 0.50079 & 0.64897 & 8.00054 & 0 & 0 & -4.72553 & 0.9251 \\
\hline 5 & 16 & 0.48175 & 0.50339 & -0.64642 & 15.99613 & 0 & 0 & 4.82479 & 0.9069 \\
\hline 6 & 32 & 0.40876 & 0.50997 & 0.63517 & 32.02910 & 0 & 0 & 1.46990 & 0.8975 \\
\hline 7 & 64 & 0.51825 & 0.52117 & 0.63090 & 66.31876 & -0.00066974 & 1 & 0.090540 & 0.9066 \\
\hline 8 & 128 & 0.51095 & 0.50345 & 0.61806 & 129.7168 & $8.62532 \mathrm{e}-5$ & 1.11225 & 0.94630 & 0.9132 \\
\hline 9 & 256 & 0.48175 & 0.49203 & 0.64200 & 266.3384 & $1.85033 \mathrm{e}-5$ & 1.52406 & 0.73950 & 0.9147 \\
\hline 10 & 512 & 0.48905 & 0.50536 & 0.61721 & 682.0366 & -0.34387 & 0.64381 & -0.35596 & 0.9291 \\
\hline 11 & 1024 & 1 & 0.5 & -0.5 & 0 & 0 & 0 & 0 & 1 \\
\hline
\end{tabular}
statistical model (3).

Table 1. Influence of a prime number on binary number in the block № 11 according to categories of binary system

The law (3) in a general view [2] allowed to prove Riemann's hypothesis [5,6].

Comparison showed the importance in blocks of sequence of prime numbers among at which serial number has only auxiliary value (we will notice that completeness of serial number will be observed only at acceptance of a complete series $\mathrm{N}=\{0,1,2,3,4,5,6, \ldots\}$ of natural numbers). Zero can't be ignored as statistical regularities won't be wine a case reliable. Therefore, the main indicator, regardless of the type series of prime numbers, it becomes statistically average (only not arithmetic-mean) binary and variable - itself a prime number.

In a column $i_{2}=1$ from Table 1 on a formula (3) we receive binary number $z_{2}=a_{1}-a_{2}=0.5-(-0.5)=1$.

The same ratio will be for a column $i_{2}=11$. Then for the block № 11 all points on columns of the first and 11th categories of binary notation will be equal $z_{2}=1$. Consistently considering all blocks of a number of prime numbers it is possible to show that for the first column (the right border of binary record of prime numbers) the condition $z_{2}=1$ will be met on all infinite half shaft of noncritical prime numbers $a(n)=(3, \infty)$. In process of growth of value of a prime number the left border will shift abruptly from block to block [2,4].

\section{Geometry of Binary Number}

On model (3) for different categories $i_{2}$ of binary number on the block of prime numbers № 11 schedules are constructed. Riemann's critical line is located on the second vertical [2]. If not to consider the first and last categories of binary system, according to Table 1 most close to a rational root of 1/2 Zeta-functions of Riemann on material values there is a category $i_{2}=2$ (Figure 1 ). Dispersion $S=0.00000676$ very small therefore the coefficient of correlation $r$ is almost equal to unit. Then it is possible to claim that the vertical $i_{2}=2$ of decomposition of a number of prime and whole prime numbers in binary numbers becomes Riemann's critical line.

From Table 1 data for the block № 11 we receive statistical model (Figure 1 ) of a look

$$
z_{2}=1 / 2-1 / 2 \cos (\pi a(n) / 2-1.59217)
$$

in which a real number 0,5 replaced with fractional number $1 / 2$. About increase in power of a number of prime numbers there will be an aspiration $1,59217 \rightarrow \pi / 2$ and therefore the formula (4) receives a type of the law

$$
z_{2}=\frac{1}{2}-\frac{1}{2} \cos \left(\frac{\pi}{2} a(n)-\frac{\pi}{2}\right)
$$




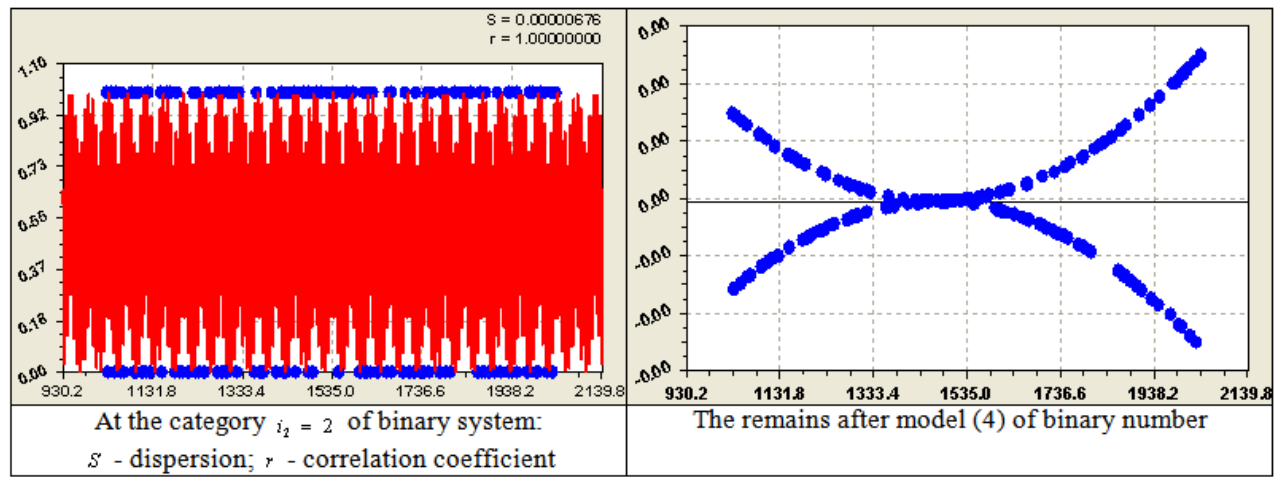

Figure 1. Schedules of statistical model of binary number on the second category

The remains receive almost zero values, but have accurate geometry of distribution.

For the category $i_{2}=3$ of binary number the equation is received (Figure 2)

$$
z_{2}=1 / 2-0.70711 \cos (\pi a(n) / 4-1.57080)
$$

which at the big power of a number of prime numbers will be transformed to a look

$$
z_{2}=\frac{1}{2}-\frac{\sqrt{2}}{2} \cos \left(\frac{\pi}{4} a(n)-\frac{\pi}{2}\right)
$$
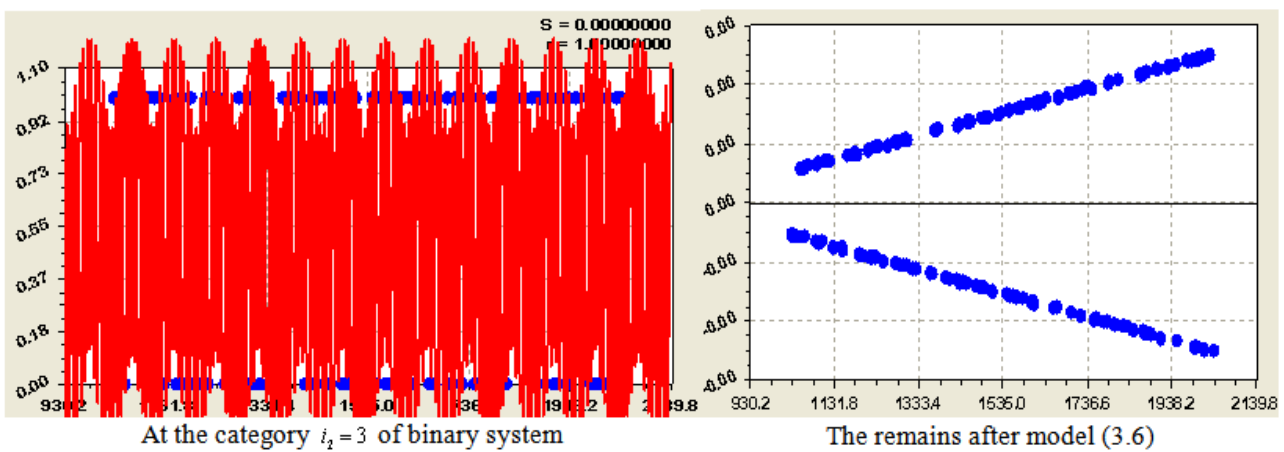

Figure 2. Schedules of statistical model of binary number on the third category of binary notation

Though the remains after a formula (6) come nearer to zero, however, as show schedules in Figure 2, the error of model increases with increase in value of a prime number. Therefore there have to be any new components to model (6).

For the category $i_{2}=4$ of binary number at the 11th block of the PN the formula is received (Figure 3)

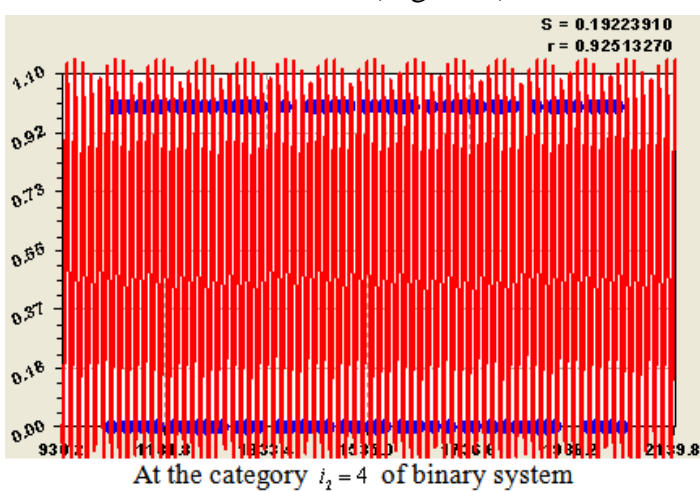

$z_{2}=0.50079-0.64897 \cos (\pi a(n) / 8.00054+4.72553)(8)$

in which values of parameters are already far from the valid root $1 / 2$.

Thus, only the second vertical becomes Riemann's critical line.

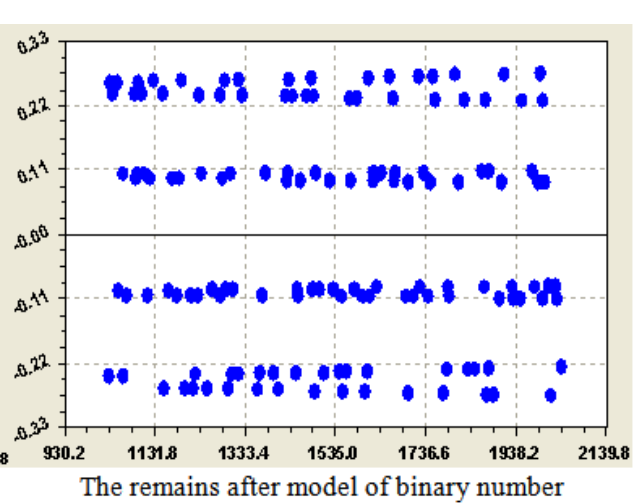

Figure 3. Schedules of statistical model of binary number on the fourth category of binary system

\section{Options of structure of the whole prime numbers (WPN)}

Blocks are allocated through transformation simple number and the whole prime numbers from decimal system in a binary numeral system.
For a number of the whole prime numbers of any power structure options are possible:

1. a symmetric finite-dimensional number of WPN with the center (is painted over) symmetry

\begin{tabular}{|c|c|c|c|c|c|c|c|c|c|c|c|c|c|c|c|}
\hline$Z$ & -7 & -6 & -5 & -4 & -3 & -2 & -1 & 0 & 1 & 2 & 3 & 4 & 5 & 6 & 7 \\
\hline$P_{Z}$ & -13 & -11 & -7 & -5 & -3 & -2 & -1 & 0 & 1 & 2 & 3 & 5 & 7 & 11 & 13 \\
\hline
\end{tabular}


2. a symmetric finite-dimensional number of WPN without the symmetry center

\begin{tabular}{|c|c|c|c|c|c|c|c|c|c|c|c|c|c|c|c|}
\hline$Z$ & -7 & -6 & - & - & - & & & & & & 3 & 4 & 5 & 6 & 7 \\
\hline$P_{Z}$ & - & - & - & - & - & & & & & & 3 & 5 & 7 & 11 & 13 \\
\hline
\end{tabular}

3. asymmetrically to the left or to the right the focused finite-dimensional ranks of WPN with the symmetry center

\begin{tabular}{|c|c|c|c|c|c|c|c|c|c|c|c|c|c|c|c|}
\hline$Z$ & $\overline{9}$ & $\overline{8}$ & $\overline{7}$ & $\overline{6}$ & $\overline{5}$ & - & - & $\overline{2}$ & $\overline{1}$ & 0 & 1 & 2 & 3 & 4 & 5 \\
\hline$P_{Z}$ & $\overline{1}$ & $\begin{array}{l}- \\
1\end{array}$ & $\begin{array}{l}- \\
1 \\
3\end{array}$ & $\begin{array}{l}- \\
1\end{array}$ & $\overline{7}$ & $\overline{5}$ & 3 & $\overline{2}$ & $\begin{array}{l}- \\
1\end{array}$ & 0 & 1 & 2 & 3 & 5 & 7 \\
\hline
\end{tabular}

\begin{tabular}{|l|c|c|c|c|c|c|c|c|c|c|c|c|c|c|c|}
\hline$Z$ & - & - & - & - & - & 0 & 1 & 2 & 3 & 4 & 5 & 6 & 7 & 8 & 9 \\
\hline$P_{Z}$ & - & - & - & - & - & 0 & 1 & 2 & 3 & 5 & 7 & 1 & 1 & 1 & 1 \\
& 7 & 5 & 3 & 2 & 1 & 0 & 1 & 2 & & & & 1 & 7 & 9 \\
\hline
\end{tabular}

4. left (negative) and right (positive) asymmetric ranks of prime numbers

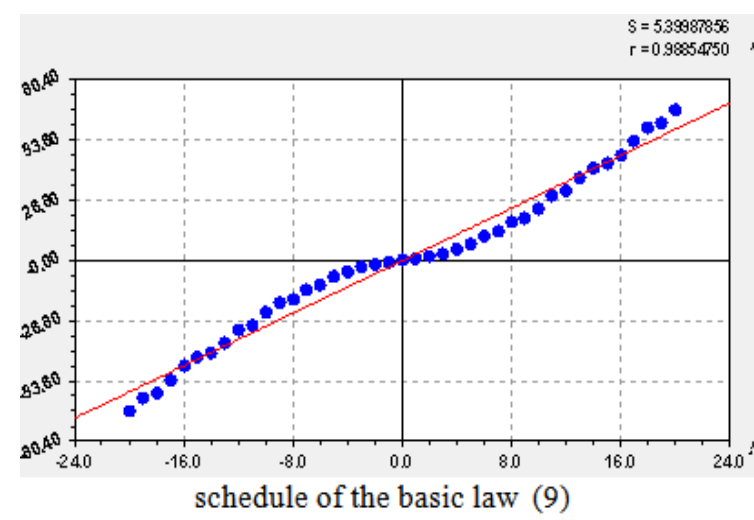

Figure 4. Schedules of a formula (9.1) distributions of 20 couples of WPN on a scale of integers

Asymmetry to the left, for example $n^{-}=20$ and

$$
P_{Z 20 л, 10 \Pi}=-1.40524+2.73831 Z
$$

$n^{+}=10$, is identified (Figure 5) a formula

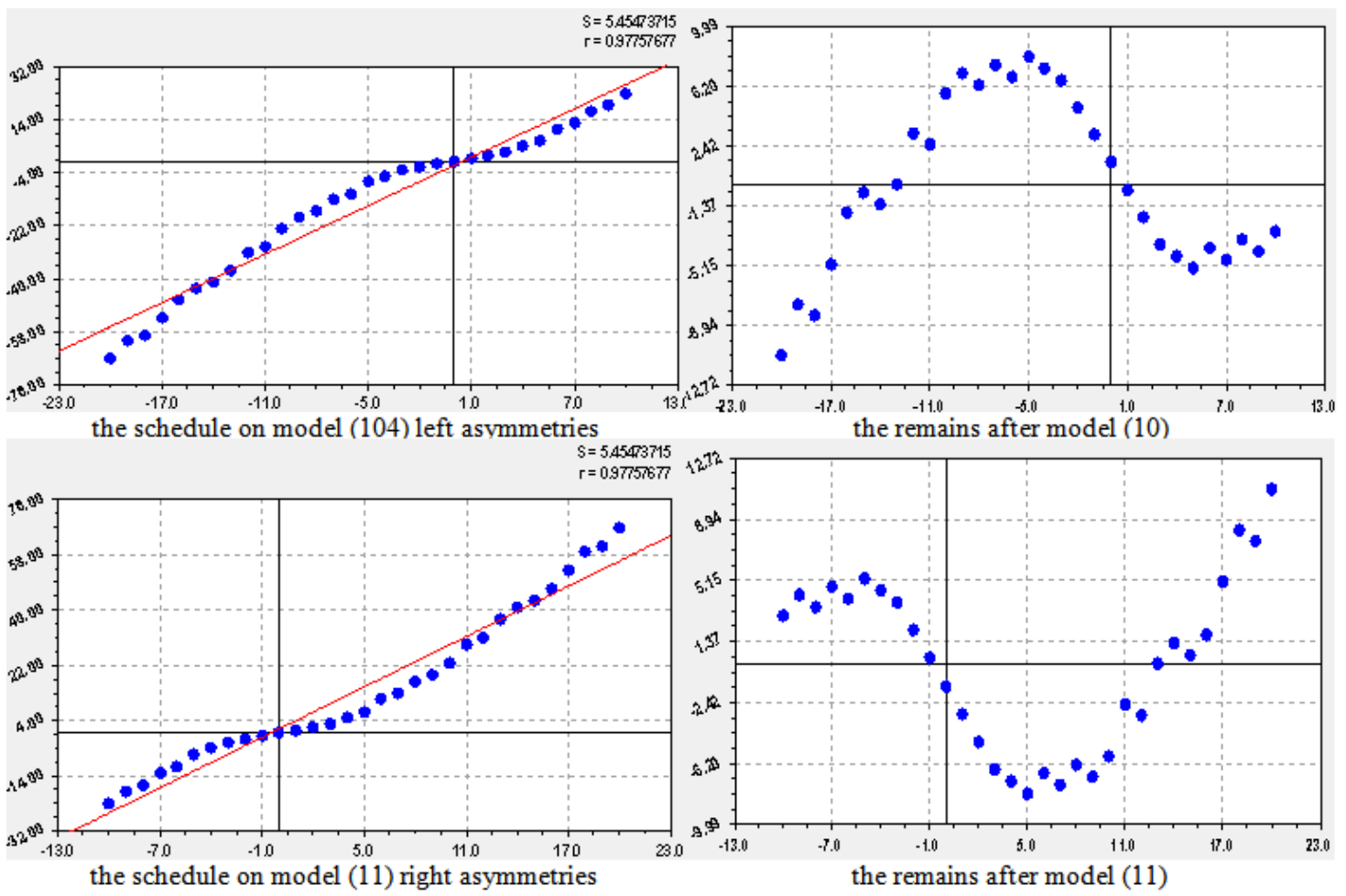

Figure 5. Schedules of distribution of asymmetric couples of WPN on a scale of integers 
At asymmetry to the right, for example $n^{-}=10$ and $n^{+}=20$, expression turns out (Figure 5)

$$
P_{\text {Z10л }, 20 \Pi}=1.40524+2.73831 Z
$$

Comparison of formulas (10) and (11) shows that the left and right asymmetry differ from each other only signs of the first component appearing in the form of the permanent member before the law of distribution of

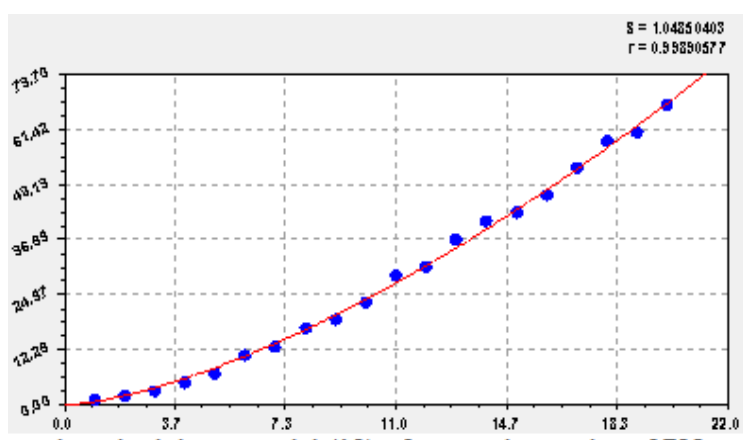

the schedule on model (12) of a complete series of PN members of a symmetric number of the whole prime numbers. We will bring asymmetry to a limit when the beginning (positive $\mathrm{PN}$ ) or the end (for negative $\mathrm{PN}$ ) are defined by the beginning of coordinates of an axis of integers.

For positive prime numbers (a complete series [2,4]) 0 , $1,2,3,5,7,11, \ldots$ (Figure 6 ) we will receive

$$
P_{+Z}=0.720694 Z_{+}^{1.515400}
$$

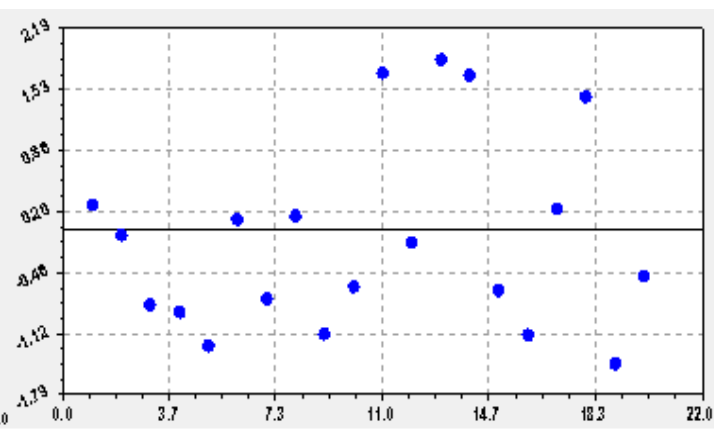

the remains after model (12)

Figure 6. Distribution of positive prime numbers on the right scale of integers

On the remains possibility of wave adaptation to 12 is visible. The program environment refuses identification because of construction of a negative number in degree. Because of property of symmetry, we receive model

$$
P_{-Z}=-0.720694\left(-Z_{-}\right)^{1.515400}
$$

For direct identification of regularities on a negative half shaft of integers it is applicable integer values of degree in the indicative law

$$
P_{Z}=a Z^{b=1,2,4,6, \ldots}
$$

By a technique $[7,8]$ we find degree in identification by search $b=1,2,4,6, \ldots$ in (14) which will give the maximum adequacy: the correlation coefficient at $b=1$ is equal $r=0.9698 ; 2-0.9834 ; 4-0.7819$.

The best is the degree equal to 2 (Figure 7), that is Gauss's theory on application of degree 2 in the law of normal distribution is confirmed $y=a \exp \left(-b(x-c)^{\wedge} 2\right)$, i.e.

$$
P_{-Z}=-0.18470 Z_{-}^{2}
$$

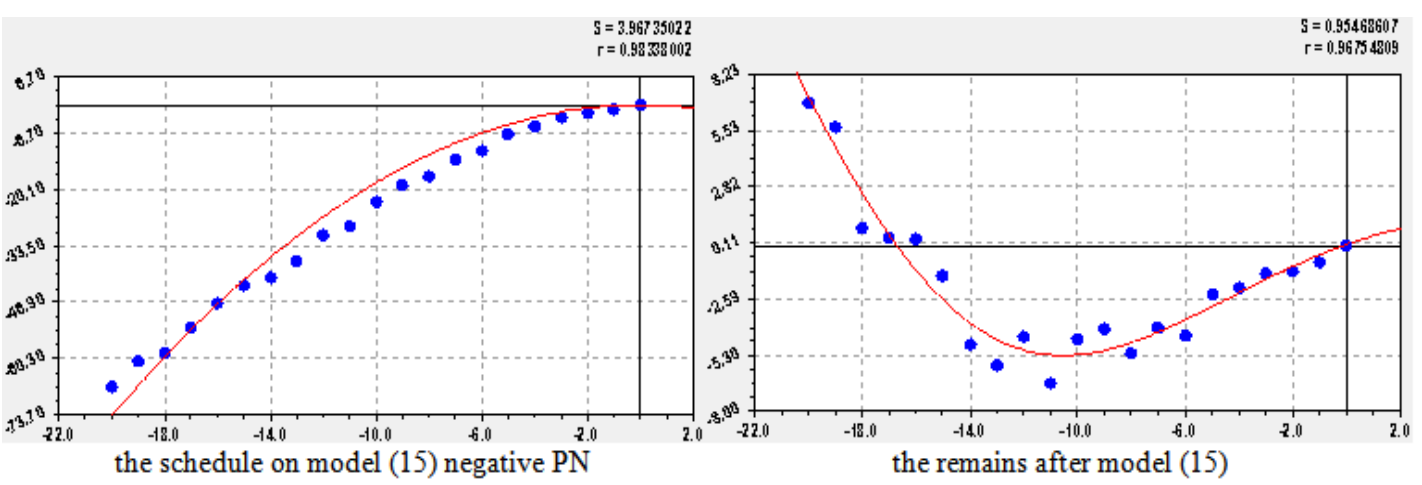

Figure 7. Distribution of negative prime numbers on the left scale of integers

Joint parametrical identification gives (Figure 8) the binomial equation
$P_{-Z 20}=-0.199339 Z_{-}^{2}$

$+1.566783 \exp \left(-0.110277 Z_{-}\right) \sin (\pi / 1.512178-0.103841)$

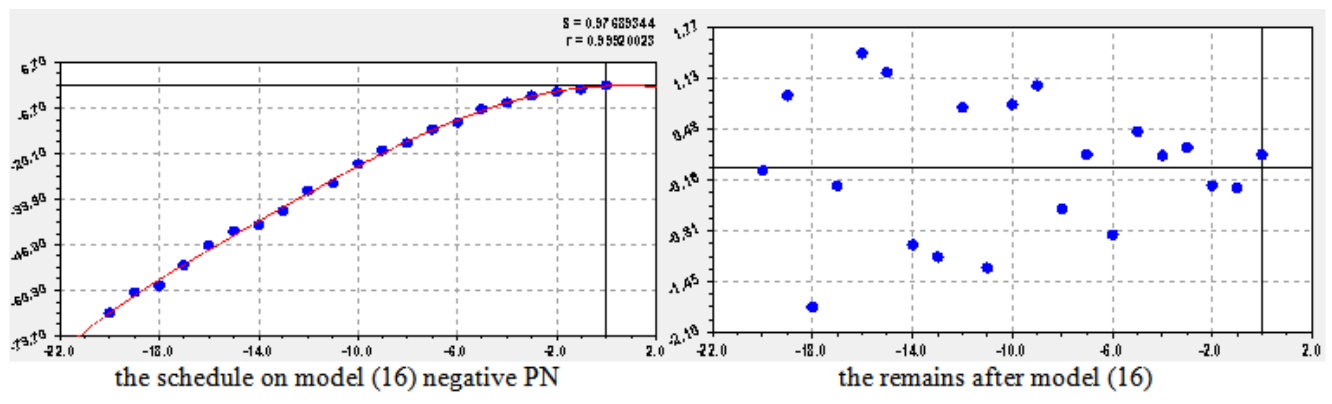

Figure 8. Distribution of 20 negative prime numbers on the left scale of integers 
Table 2. Example of decomposition of 20 couples of WPN and gain

\begin{tabular}{|c|c|c|c|c|c|c|c|c|c|c|c|c|}
\hline \multirow{4}{*}{ Integer $Z$} & \multirow{4}{*}{ Prime number $P_{Z}$} & \multirow{4}{*}{ Gain $p_{Z}$} & \multicolumn{7}{|c|}{ Category $i_{Z}^{P}$ binary } & \multicolumn{3}{|c|}{ Category $i_{Z}^{p}$} \\
\hline & & & 7 & 6 & 5 & 4 & 3 & 2 & 1 & 3 & 2 & 1 \\
\hline & & & \multicolumn{7}{|c|}{ Part $P_{i Z}=2^{\wedge}\left(i_{Z}^{P}-1\right)$} & \multicolumn{3}{|c|}{$p_{i Z}=2 \wedge\left(i_{Z}^{p}-1\right)$} \\
\hline & & & 64 & 32 & 16 & 8 & 4 & 2 & 1 & 4 & 2 & 1 \\
\hline-20 & -67 & 6 & -1 & 0 & 0 & 0 & 0 & -1 & -1 & 1 & 1 & 0 \\
\hline-19 & -61 & 2 & & -1 & -1 & -1 & -1 & 0 & -1 & & 1 & 0 \\
\hline-18 & -59 & 6 & & -1 & -1 & -1 & 0 & -1 & -1 & 1 & 1 & 0 \\
\hline-17 & -53 & 6 & & -1 & -1 & 0 & -1 & 0 & -1 & 1 & 1 & 0 \\
\hline-16 & -47 & 4 & & -1 & 0 & -1 & -1 & -1 & -1 & 1 & 0 & 0 \\
\hline-15 & -43 & 2 & & -1 & 0 & -1 & 0 & -1 & -1 & & 1 & 0 \\
\hline-14 & -41 & 4 & & -1 & 0 & -1 & 0 & 0 & -1 & 1 & 0 & 0 \\
\hline-13 & -37 & 6 & & -1 & 0 & 0 & -1 & 0 & -1 & 1 & 1 & 0 \\
\hline-12 & -31 & 2 & & & -1 & -1 & -1 & -1 & -1 & & 1 & 0 \\
\hline-11 & -29 & 6 & & & -1 & -1 & -1 & 0 & -1 & 1 & 1 & 0 \\
\hline-10 & -23 & 4 & & & -1 & 0 & -1 & -1 & -1 & 1 & 0 & 0 \\
\hline-9 & -19 & 2 & & & -1 & 0 & 0 & -1 & -1 & & 1 & 0 \\
\hline-8 & -17 & 4 & & & -1 & 0 & 0 & 0 & -1 & 1 & 0 & 0 \\
\hline-7 & -13 & 2 & & & & -1 & -1 & 0 & -1 & & 1 & 0 \\
\hline-6 & -11 & 4 & & & & -1 & 0 & -1 & -1 & 1 & 0 & 0 \\
\hline-5 & -7 & 2 & & & & & -1 & -1 & -1 & & 1 & 0 \\
\hline-4 & -5 & 2 & & & & & -1 & 0 & -1 & & 1 & 0 \\
\hline-3 & -3 & 1 & & & & & & -1 & -1 & & & 1 \\
\hline-2 & -2 & 1 & & & & & & -1 & 0 & & & 1 \\
\hline-1 & -1 & 1 & & & & & & & -1 & & & 1 \\
\hline 0 & 0 & 1 & & & & & & & 1 & & & 1 \\
\hline 1 & 1 & 1 & & & & & & & 1 & & & 1 \\
\hline 2 & 2 & 1 & & & & & & 1 & 0 & & & 1 \\
\hline 3 & 3 & 2 & & & & & & 1 & 1 & & 1 & 0 \\
\hline 4 & 5 & 2 & & & & & 1 & 0 & 1 & & 1 & 0 \\
\hline 5 & 7 & 4 & & & & & 1 & 1 & 1 & 1 & 0 & 0 \\
\hline 6 & 11 & 2 & & & & 1 & 0 & 1 & 1 & & 1 & 0 \\
\hline 7 & 13 & 4 & & & & 1 & 1 & 0 & 1 & 1 & 0 & 0 \\
\hline 8 & 17 & 2 & & & 1 & 0 & 0 & 0 & 1 & & 1 & 0 \\
\hline 9 & 19 & 4 & & & 1 & 0 & 0 & 1 & 1 & 1 & 0 & 0 \\
\hline 10 & 23 & 6 & & & 1 & 0 & 1 & 1 & 1 & 1 & 1 & 0 \\
\hline 11 & 29 & 2 & & & 1 & 1 & 1 & 0 & 1 & & 1 & 0 \\
\hline 12 & 31 & 6 & & & 1 & 1 & 1 & 1 & 1 & 1 & 1 & 0 \\
\hline 13 & 37 & 4 & & 1 & 0 & 0 & 1 & 0 & 1 & 1 & 0 & 0 \\
\hline 14 & 41 & 2 & & 1 & 0 & 1 & 0 & 0 & 1 & & 1 & 0 \\
\hline 15 & 43 & 4 & & 1 & 0 & 1 & 0 & 1 & 1 & 1 & 0 & 0 \\
\hline 16 & 47 & 6 & & 1 & 0 & 1 & 1 & 1 & 1 & 1 & 1 & 0 \\
\hline 17 & 53 & 6 & & 1 & 1 & 0 & 1 & 0 & 1 & 1 & 1 & 0 \\
\hline 18 & 59 & 2 & & 1 & 1 & 1 & 0 & 1 & 1 & & 1 & 0 \\
\hline 19 & 61 & 6 & & 1 & 1 & 1 & 1 & 0 & 1 & 1 & 1 & 0 \\
\hline 20 & 67 & 4 & 1 & 0 & 0 & 0 & 0 & 1 & 1 & 1 & 0 & 0 \\
\hline
\end{tabular}


Here the first negative member shows square function, and the second - recession on amplitude at the constant period of fluctuation $2 \times 1.51218=3.02436$.

\section{Binary Number of Symmetric Ranks}

Data for identification are provided in Table 2.

Influence of the whole prime numbers $P_{Z 20}$ on binary number $z_{2}$ on the vertical $i_{Z}^{P}=2$ goes on the look equation

$$
z_{2}=-0.5 \sin \left(\pi P_{Z 20-3} / 2\right)
$$

Here three WPN $(-1,0,1)$ of a core of the center of symmetry were excluded because in the second vertical in these cages there are trivial zero.

The coefficient of correlation (17) is equal slightly more than 0,7: the beginning is stirred with a prime number Gauss- Riemann 2 rows $(2,3,5,7, \ldots)$.

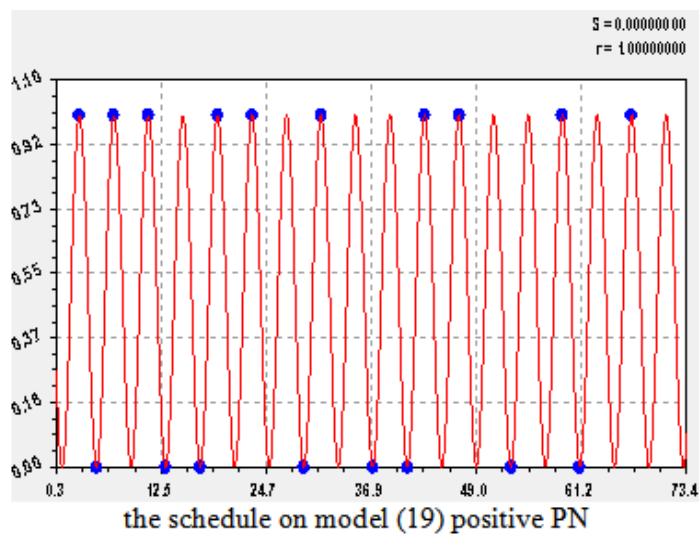

After exclusion of WPN center of symmetry in two symmetric about the axis $Z$ of noncritical series $\pm(3,5$, $7,11, . .$.$) , to obtain a formula of the form$

$$
z_{2}=\cos \left(\pi P_{Z 20-5} / 2+0.58903\right)
$$

The coefficient of correlation became equal 0,7454 . Then it turns out that trivial zero always divide verticals into the isolated parts. Therefore it is necessary to model at once in parts noncritical half of ranks $P_{Z= \pm(3,5,7,11, \ldots)}$

(Figure 9):

- for positive prime numbers

$$
z_{2}=\frac{1}{2}-\frac{1}{2} \sin \left(\frac{\pi}{2} P_{Z=3, \ldots}\right)
$$

- for negative prime numbers

$$
z_{2}=-\frac{1}{2}-\frac{1}{2} \sin \left(\frac{\pi}{2} P_{Z=-3, \ldots}\right)
$$

Thus, Riemann's hypothesis of a root $1 / 2$ is proved and on a number of WPN.

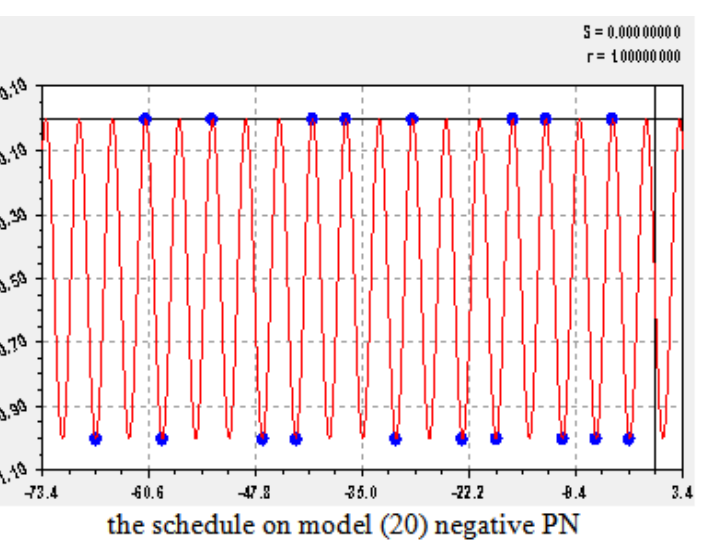

Figure 9. Distribution of binary number on the right and left scale of the whole noncritical prime numbers

With increase in the category of number $\pi$ more than 18 [8] remains will be much less 1e-12.

Influence of the WPN in a symmetric row $P_{Z 20}$ to a binary number $z_{2}$ part of the increase vertically $i_{Z}^{p}=2$ driven by empirical formula.

$$
z_{2}=0.5-0.5 \sin \left(\pi P_{Z 20} / 1.73942\right)
$$

Then it was necessary to exclude six WPN values (-3,$2,-1,0,1,2)$ because of existence of trivial zero. A positive number of $\mathrm{PN}$ begins with 3 . And a negative number of PN begins with Figure 5 therefore even in a symmetric number of WPN asymmetry in binary quantification of a gain is observed.

Further we will show influence of a gain of WPN (row $\left.p_{Z 20}\right)$ on binary number $z_{2}$ on a vertical $i_{Z}^{p}=2$ :

$$
z_{2}=0.5-0.5 \cos \left(\pi p_{Z 20} / 2.12144\right)
$$

Because of an exception of six points influence of a gain on the second vertical of binary representation turns out model (Figure 10) of a look

$$
z_{2}=0.5-0.5 \cos \left(\pi p_{Z 20-6} / 2\right)
$$
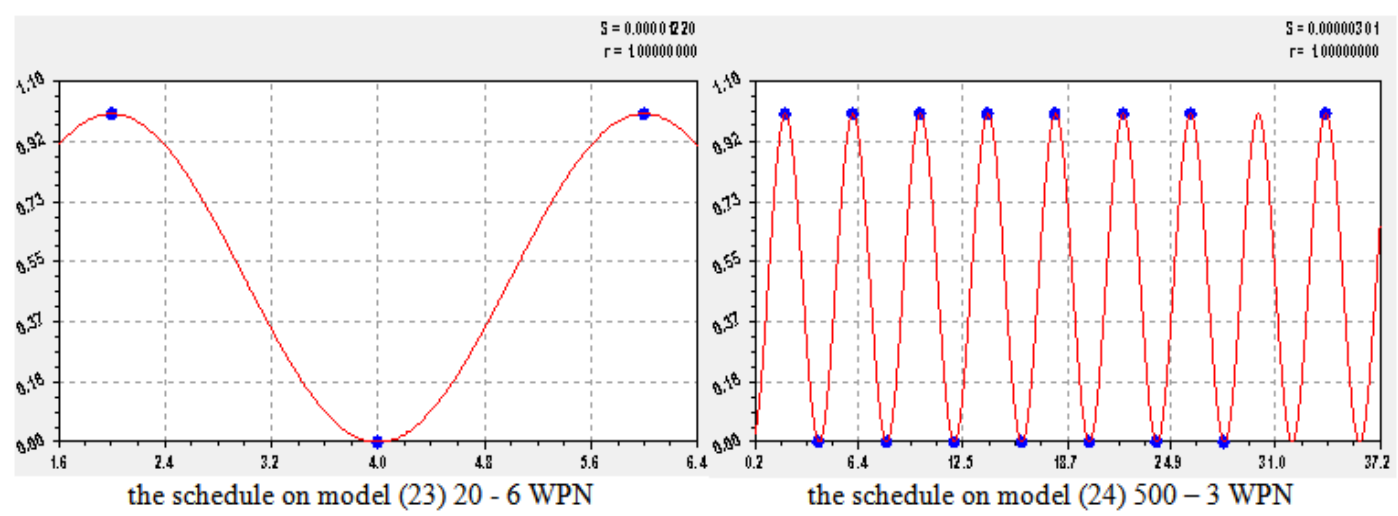

Figure 10. Distribution of binary number of noncritical whole prime numbers from a gain of WPN 
For the massif from $500-3$ noncritical values of prime numbers (Figure 10) the formula is given

$$
z_{2}=0.5-0.5 \cos \left(\pi P_{Z \pm(500-3)} / 2\right)
$$

Both half-rows of WPN if to exclude the symmetry center, are identical.
Thus, Riemann's hypothesis is proved and on a number of the whole prime numbers.

\section{Block Structure of a Number of WPN}

Table 3. Length, left and right border of blocks of the whole prime numbers

\begin{tabular}{|c|c|c|c|c|c|c|c|c|c|c|}
\hline \multirow[b]{2}{*}{$\begin{array}{l}\text { Categor } \\
\text { y } i\end{array}$} & \multirow[b]{2}{*}{ $\pm 2^{i-1}$} & \multicolumn{3}{|c|}{ Left border } & \multicolumn{3}{|c|}{ Right border } & \multicolumn{3}{|c|}{ Length of the block of binary numbers } \\
\hline & & $\pm P_{N}^{\prime}$ & $\begin{array}{c}n_{ \pm}^{\prime} \\
\text { piece }\end{array}$ & $2 P_{N}^{\prime} /\left(n_{ \pm}^{\prime}-1\right)$ & $\pm P_{N}^{\prime \prime}$ & $\begin{array}{r}n_{ \pm}^{\prime \prime}, \\
\text { piece }\end{array}$ & $2 P_{N}^{\prime \prime} /\left(n_{ \pm}^{\prime \prime}-1\right)$ & $\Delta P_{N}=P^{\prime \prime}-P^{\prime}$ & $\Delta n=n^{\prime \prime}-n^{\prime}$ & $2 \Delta P_{N} / \Delta n$ \\
\hline 0 & $1 / 2$ & $1 / 2$ & 1 & - & $1 / 2$ & 1 & - & 0 & 0 & - \\
\hline 1 & 1 & 1 & 3 & 1 & 1 & 3 & 1 & 0 & 0 & - \\
\hline 2 & 2 & 2 & 5 & 1 & 2 & 5 & 1 & 0 & 0 & - \\
\hline 3 & 4 & 3 & 7 & 1 & 3 & 7 & 1 & 0 & 0 & - \\
\hline 4 & 8 & 5 & 9 & 1.2500 & 7 & 11 & 1.4000 & 2 & 2 & 2 \\
\hline 5 & 16 & 11 & 13 & 1.8333 & 13 & 15 & 1.8571 & 2 & 2 & 2 \\
\hline 6 & 32 & 17 & 17 & 2.1250 & 31 & 25 & 2.5833 & 14 & 8 & 3.5000 \\
\hline 7 & 64 & 37 & 27 & 2.8462 & 61 & 39 & 3.2105 & 24 & 12 & 4.0000 \\
\hline 8 & 128 & 67 & 41 & 3.3500 & 127 & 65 & 3.9688 & 60 & 24 & 5.0000 \\
\hline 9 & 256 & 131 & 67 & 3.9697 & 251 & 111 & 4.5636 & 120 & 44 & 5.4545 \\
\hline 10 & 512 & 257 & 113 & 4.5893 & 509 & 197 & 5.1939 & 252 & 84 & 6.0000 \\
\hline 11 & 1024 & 521 & 199 & 5.2626 & 1021 & 347 & 5.9017 & 500 & 148 & 6.7568 \\
\hline 12 & 2048 & 1031 & 349 & 5.9253 & 2039 & 621 & 6.5774 & 1008 & 272 & 7.4118 \\
\hline 13 & 4096 & 2053 & 623 & 6.6013 & 4093 & 1131 & 7.2442 & 2040 & 508 & 8.0315 \\
\hline 14 & 8192 & 4099 & 1133 & 7.2420 & 8191 & 2059 & 7.9602 & 4092 & 926 & 8.8380 \\
\hline
\end{tabular}

In any finite-dimensional number of prime numbers, including and the whole prime numbers, blocks are allocated through transformation, that is quantization, prime numbers from decimal system in a binary numeral system. We will divide a number of WPN on blocks (categories $i$ ) of binary notation (Table 3) to power by computing opportunities of the program CurveExpert-1.40 environment.

All parameters from Table 3 are identified by the law of indicative growth. The root $1 / 2$ automatically turns out under a condition $i=0$. The border between blocks of prime numbers precisely is defined by the corresponding part from a decimal numeral system.

Exactly there are jumps of the second type (the first type of jump is in blocks on so-called primary gain) among between values at adjacent prime numbers, not clear to mathematics.

This boundary can be approximately replaced (Figure 11) by the equation

$$
\pm 2^{i-1} \approx 1 / 2+5.04767 \cdot 10^{-7} i^{8.90632}
$$

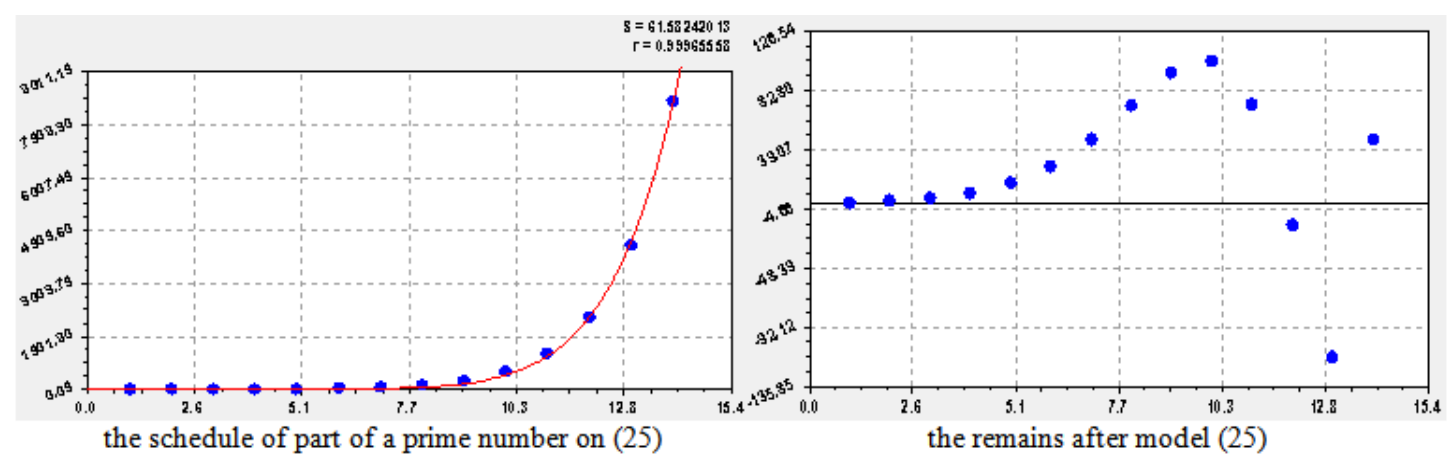

Figure 11. Influence of the category of a binary numeral system on part of PN from a decimal numeral system

Each block of PN [2,4] is defined by the left and right borders, and also block length along a number of prime numbers. At all formulas the remains are by the form similar to points in Figure 11.
For comparison in Figure 12 schedules of the left and right border are given. It is visible that the rational root of $1 / 2$ Zeta-functions of Riemann is in a formula of prime numbers from the category of a binary numeral system. 

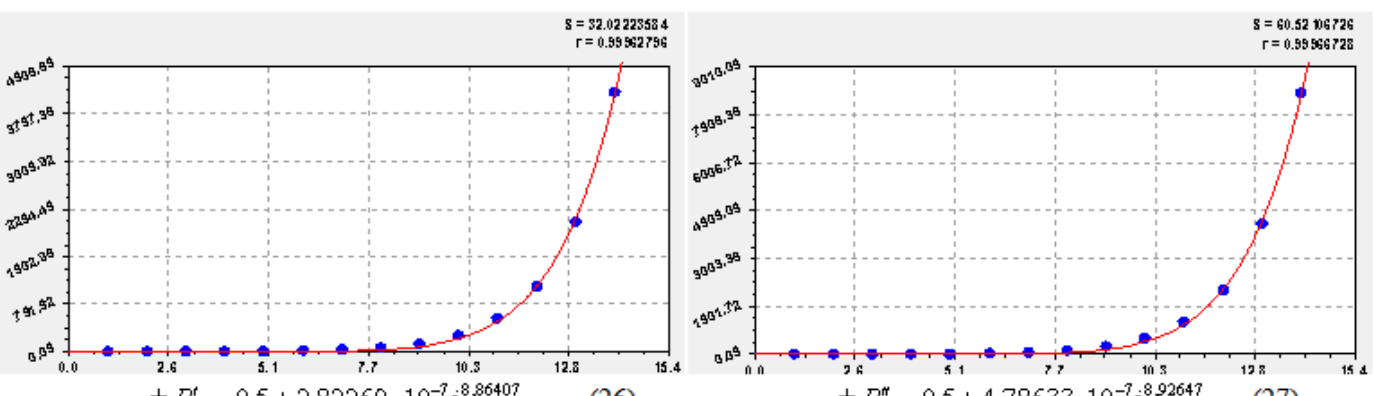

$\pm P_{N}^{t}=0,5+2,82269 \cdot 10^{-7} i^{8,86407} \quad(26)$

$\pm P_{N}^{\mathrm{N}}=0,5+4,78633 \cdot 10^{-7} i^{8,92647}$

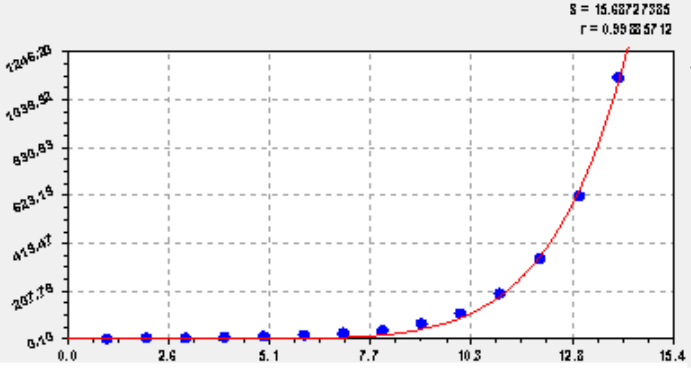

$n_{ \pm}^{t}=1+3,88361 \cdot 10^{-6} i^{7,3006}$

(28)

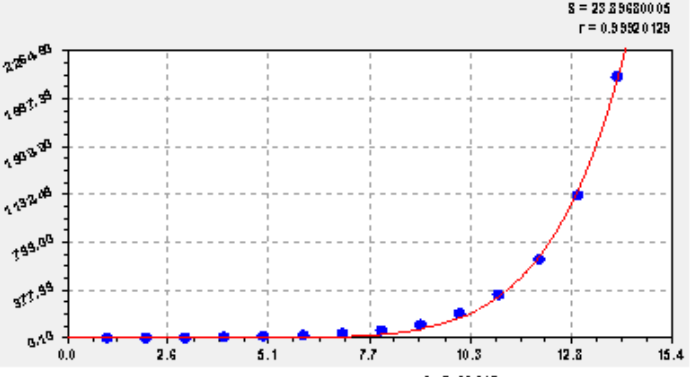

$n_{ \pm}^{\prime \prime}=1+4,81911 \cdot 10^{-6} i^{7,52643}$

(29)

Figure 12. Parameters of the left and right border of blocks of prime numbers

Harder change average in Prime numbers to the left and right border blocks (Figure 13).

In the beginning on trends from the category of a binary numeral system formulas are received:
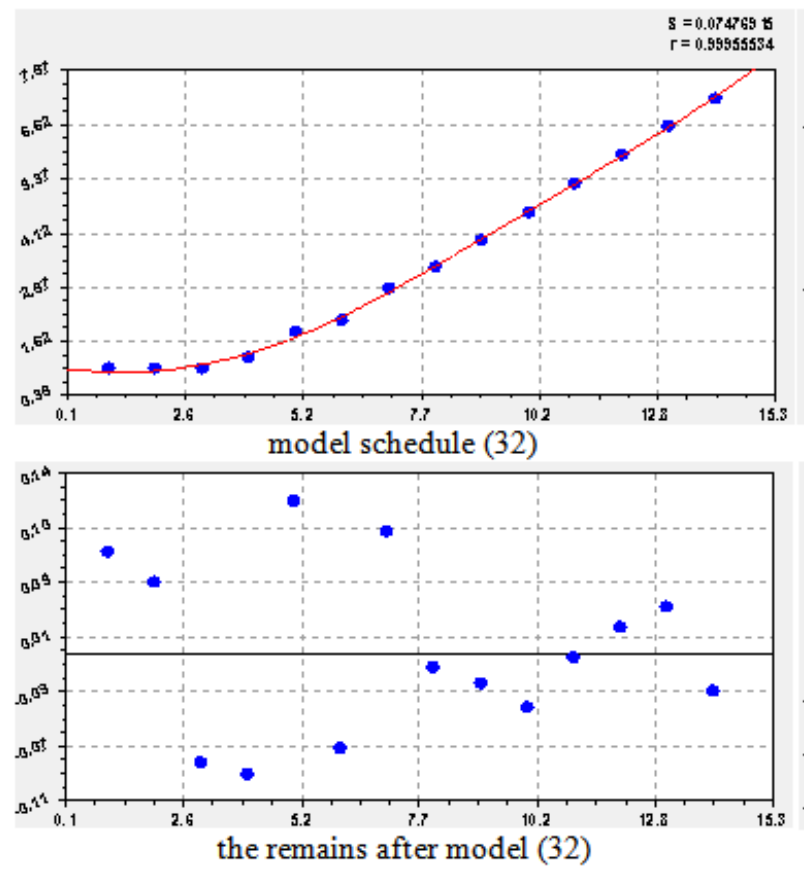

Figure 13. Average values of intervals of prime numbers on borders of blocks of binary decomposition of WPN
Then there was a trend addition with a simple sinusoid on expressions:

$$
\begin{aligned}
& 2 P_{N}^{\prime} /\left(n_{ \pm}^{\prime}-1\right)=1+0.043455 i^{1.88851} \\
& -0.26761 \sin (\pi i / 6.68984) \\
& 2 P_{N}^{\prime \prime} /\left(n_{ \pm}^{\prime \prime}-1\right)=1+0.060123 i^{1.81554} \\
& -0.35172 \sin (\pi i / 6.18813)
\end{aligned}
$$

The remains after formulas (32) and (33) show possibility of additional wave function with a decreasing

$$
\begin{aligned}
& 2 P_{N}^{\prime} /\left(n_{ \pm}^{\prime}-1\right)=1+0.044434 i^{1.88785} \\
& 2 P_{N}^{\prime \prime} /\left(n_{ \pm}^{\prime \prime}-1\right)=1+0.069727 i^{1.75861}
\end{aligned}
$$
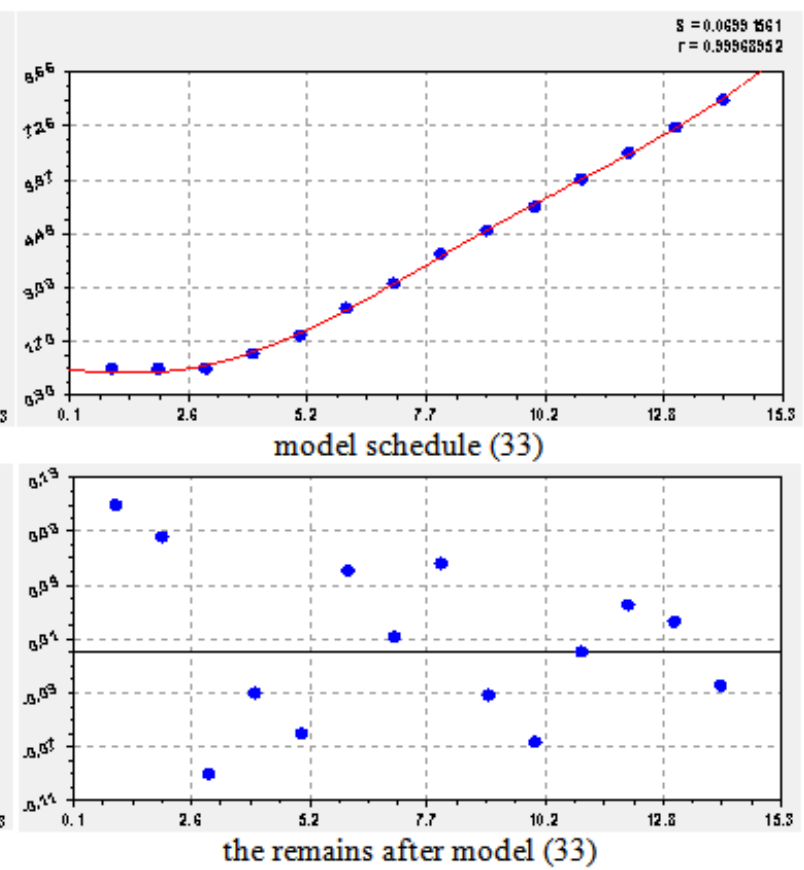

amplitude. Apparently from Table 3 data, length of the block begins only with $i=4$. Therefore prime numbers start being ordered not from the symmetry center, and is much farther from it.

For three parameters (without values to $i=3$ ) are received by equation (Figure 14):

$$
\begin{gathered}
\Delta P_{N}=P^{\prime \prime}-P^{\prime}=2.02671 \cdot 10^{-7} i^{8.98930} \\
\Delta n=n^{\prime \prime}-n^{\prime}=1.35859 \cdot 10^{-6} i^{7.70459} \\
2 \Delta P_{N} / \Delta n=-0.90145+0.69605 i
\end{gathered}
$$



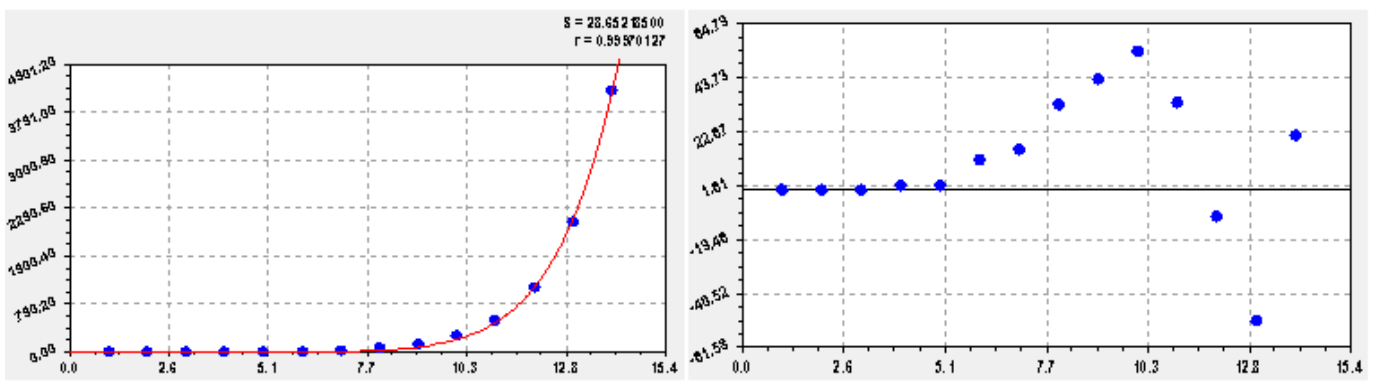

length of the block of binary numbers

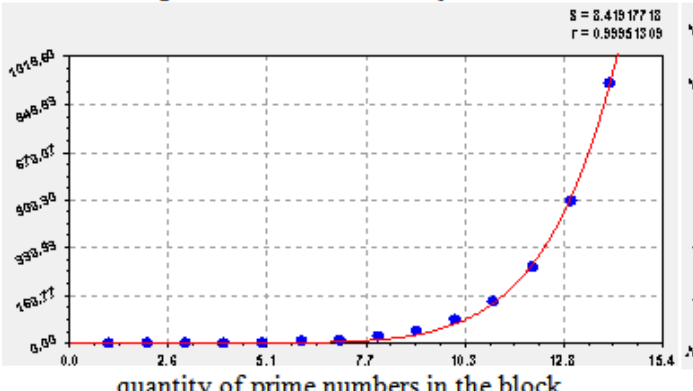

quantity of prime numbers in the block
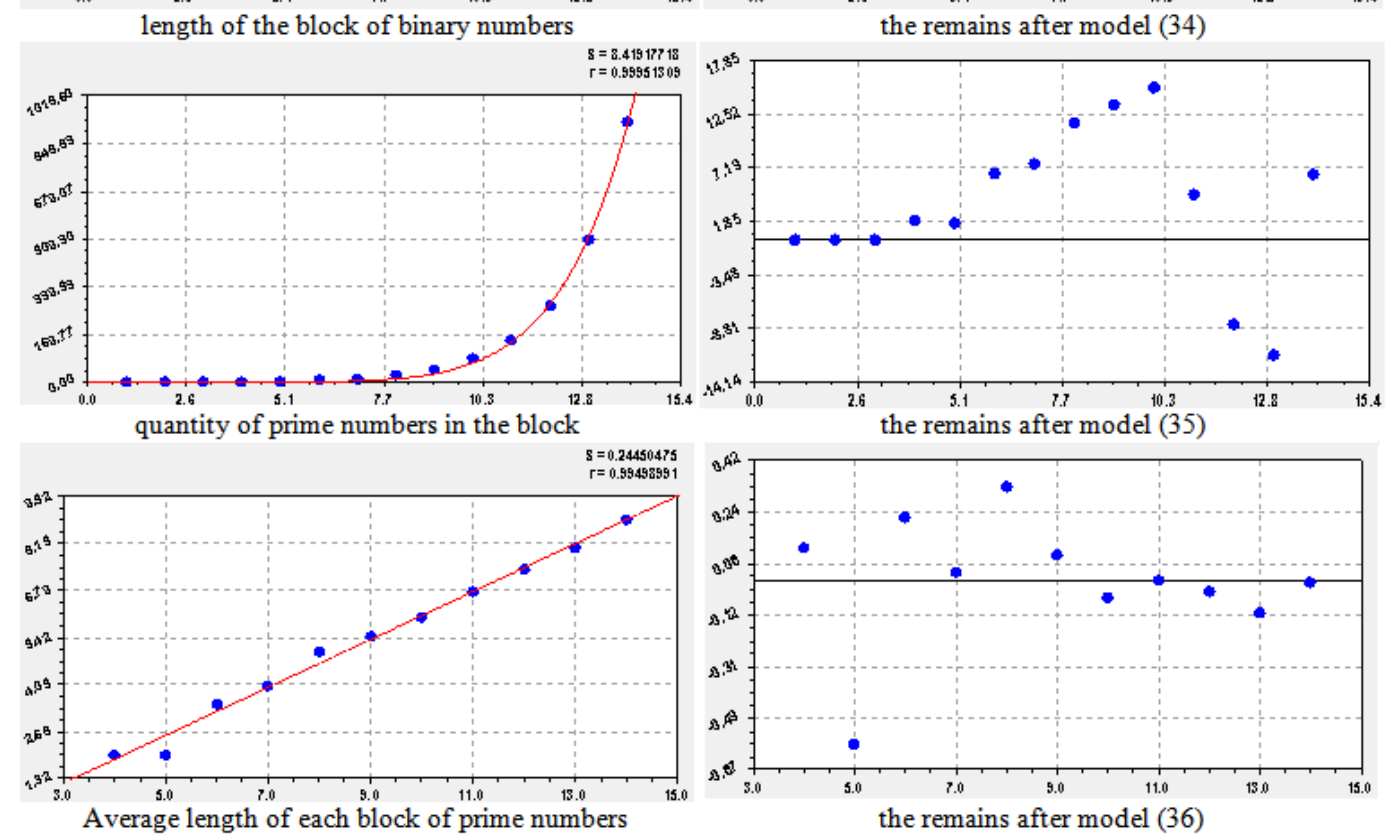

Figure 14. Parameters of length of blocks at binary representation of a number of prime numbers

Average length of the block of binary representation of the whole prime numbers becomes the best parameter for an assessment of a number of prime numbers. And it is enough to spread out only the right positive side of this row. Apparently from Figure 14, the remains after $i \geq 10$ gradually come nearer to zero, and length of each block increases further in proportion to the category of a binary numeral system.

Then it turns out that the power of a number of PN and / or WPN convenient to assume the blocks, than on existence of prime numbers in categories of a decimal numeral system. It is one more law of distribution of prime numbers.

\section{The real part $1 / 2$}

From the Internet it is known: "And here Riemann's well-known hypothesis that real part of a root always is equal in accuracy $1 / 2$, still is proved by nobody though its proof would have for the theory of prime numbers extremely importance".

The equation (3) and its special cases prove that not only the real part of a root is equal $1 / 2$.

There are also other interesting mathematical results. For example, in formulas (5) and (7) expression before function of a cosine also precisely equally 1/2. Emergence of number of space turns the equation (5) into a signal in the form of symmetric wavelet with a constant amplitude of $\pm 1 / 2$.

Zeta-functions of Riemann has zero in negative numbers, multiple 2 . But these tables 1 show that frequency rate is equal $2^{i^{-1}}$. Then according to Riemann it turns out that $2^{i_{2}-1}=2$ only under a condition $i_{2}=2$.

We will notice also that in Zeta-functions of Riemann in complex variables sine function is accepted, but the cosine is better for real numbers as allows not to pay attention to signs in expression under trigonometric function. The cosine works in both quadrants at a number of natural numbers $(0,1,2, \ldots, \infty)$. Therefore this trigonometric function will be successful further in studying of ranks of prime numbers.

\section{Conclusion}

The hobby for factorization didn't allow mathematics to apply binary system to the analysis not only multipliers of any prime number, but also quantized in a binary numeral system of a composed from any natural and prime number. Decomposition on composed of a binary numeral system gives advantages on presentation.

For infinite-dimensional series of prime numbers we have levels of quantization or area of categories of binary system. As a result we receive two types of zero - trivial and nontrivial. The first are located at the left to a vertical in each block in the form of unit. Nontrivial zero settle inside two columns with units, and the left column 1 moves on blocks from right to left in process of increase in a prime number.

The category of a prime number of a binary numeral system plays a crucial role for decomposition of a known 
prime number and forecasting of the next unknown prime number.

Comparison showed importance in blocks of only the sequence of prime numbers, and serial number has only auxiliary value. Completeness of serial number will be observed only at acceptance of a complete series $\mathrm{N}=\{0,1,2,3,4,5,6, \ldots\}$ of natural numbers. Zero can't be ignored on abscissa axis. Therefore, the main indicator becomes the average (only not arithmetic-mean) binary number, and the variable is itself a prime number.

Consistently considering all blocks of a number of prime numbers it is proved that for the first column (the right border of binary record of prime numbers) the condition $z_{2}=1$ will be met on all infinite half shaft of prime numbers of a noncritical row. The left border will be displaced from the block to the block in process of growth of value of a prime number.

In process of growth of the category of a binary numeral system across a row in the block № 11 from a traditional number $a(n)=(1031,2039)$ of prime numbers there is a decrease in adequacy of statistical model.

\section{Reference}

[1] P.M. Mazurkin, "Wavelet Analysis of a Number of Prime Numbers." American Journal of Numerical Analysis, vol. 2, no. 2 (2014): 29-34

[2] P.M. Mazurkin. Patterns of primes. Germany : Palmarium Academic Publishing, 2012. 280 p.

[3] P.M. Mazurkin, "Stable Laws and the Number of Ordinary." Applied Mathematics and Physics, vol. 2, no. 2 (2014): 27-32.

[4] P.M. Mazurkin, "Series Primes in Binary." American Journal of Applied Mathematics and Statistics, vol. 2, no. 2 (2014): 60-65.

[5] P.M. Mazurkin, "Proof the Riemann Hypothesis." American Journal of Applied Mathematics and Statistics, vol. 2, no. 1 (2014): 53-59.

[6] P.M. Mazurkin, "Increment Primes.” American Journal of Applied Mathematics and Statistics, vol. 2, no. 2 (2014): 66-72.

[7] P.M. Mazurkin, "Identification of statistical stable patterns." SCIENCE AND WORLD. International scientific journal, № 3 (3), 2013. p. 28-33.

[8] P.M. Mazurkin, A.S. Filonov, "Mathematical modeling. Identification univariate statistical regularities: tutorial." YoshkarOla, Mari State Technical University, 2006. 292 p. 